\title{
Severe atherosclerotic changes, including aortic occlusion, associated with hyperhomocysteinaemia and antiphospholipid antibodies
}

\author{
P E Spronk, E H Overbosch, N H Schut
}

\begin{abstract}
Three patients are described with severe systemic atherosclerosis, including aortic occlusion, in the presence of a spectrum of risk factors, including hypercholesterolaemia, hypertension, a positive family history of cardiovascular problems, and hyperhomocysteinaemia. In all three patients high levels of anticardiolipin antibodies were found. The possible pathogenic role of antiphospholipid antibodies in atherosclerosis in the context of hyperhomocysteinaemia in these patients is discussed.
\end{abstract}

(Ann Rheum Dis 2001;60:699-701)

We describe three patients with severe systemic atherosclerosis including one with aortic occlusion. High levels of antiphospholipid antibodies (aPLs) and hyperhomocysteinaemia were present in all three patients, who were also heavy smokers. To our knowledge, this is the first report in which the relation between severe atherosclerosis, aPLs, and hyperhomocysteinaemia is discussed. There has been much debate about the possible pathophysiological role of aPLs in atherosclerosis. The presence of these autoantibodies and other known atherogenic factors in our patients is discussed.

\section{Case reports}

PATIENT 1

A 41 year old man was seen at our outclinic because of hypertension. He smoked 10 cigarettes a day with a history of 25 pack years. His father also had had hypertension and angina pectoris. Apart from the hypertension (blood pressure $220 / 110 \mathrm{~mm} \mathrm{Hg}$ ), a physical examination was unremarkable. In particular, no bruits were heard over the renal arteries. The patient was taking amlodipine $5 \mathrm{mg}$ /day and atenolol $100 \mathrm{mg} /$ day. Ultrasound examination of his abdomen showed a smaller left kidney (12.7 cm right side; $9.2 \mathrm{~cm}$ left side), which had shrunk since a similar investigation one year previously $(12.3 \mathrm{~cm})$. As renal artery stenosis was suspected, angiography was performed.

Injection of contrast into the descending thoracic aorta showed that the aorta was occluded. Huge intercostal arteries feeding collaterals over the abdominal wall to the pelvic and leg arteries were found. Severe atheromatous plaques, an occluded left renal artery, a partially $(70 \%)$ occluded right renal artery, an occluded coeliac trunk, and partially (50\%) occluded superior mesenteric artery were found (fig 1).

In view of these findings, additional laboratory tests were carried out (table 1). Homocysteine levels were very high, and folate levels were slightly decreased. After folate treatment (5 mg once a day), homocysteine levels normalised (although still in the higher range) as measured by the methionine loading test. Anticardiolipin antibodies of both $\operatorname{IgM}$ and IgG class were present at high levels (repeated testing), whereas lupus anticoagulant and Venereal Disease Research Laboratory results were negative. High density lipoprotein (HDL) cholesterol concentration was decreased and triglycerides were increased. Treatment with coumarins was started.

The risk of complications during or after extensive vascular surgery were explained to the patient. He declined the offer of surgical intervention. So far (one year later), he is doing well.

\section{PATIENT 2}

This 50 year old man with intermittent claudication presented at the outclinic for evaluation. His walking distance had decreased to $200 \mathrm{~m}$. He had a smoking history of 50 pack years. Various family members had had vascular problems before the age of 60: his mother had undergone coronary artery bypass grafting three times; his sister had peripheral vascular disease requiring several angioplasties; his daughter has hyperhomocysteinaemia without

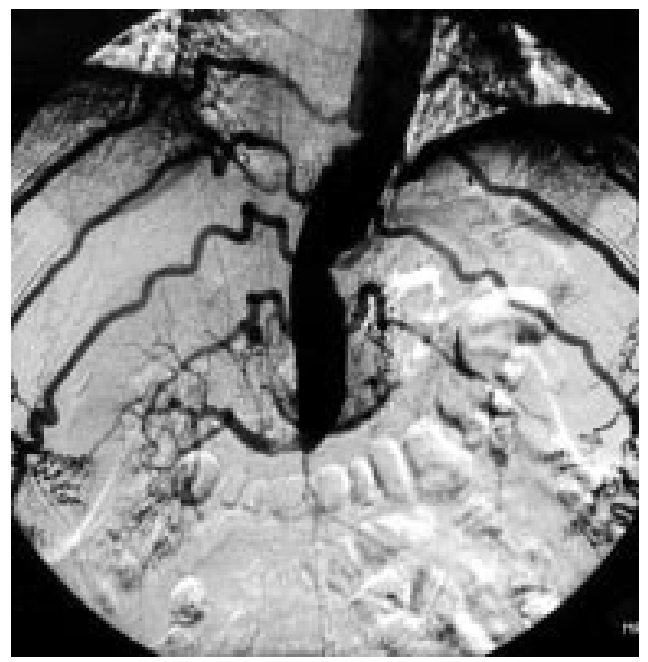

Figure 1 Angiograph showing an occluded aorta. Huge intercostal arteries feeding collaterals over the abdominal wall to the pelvic and leg arteries can be seen. Severe atheromatous plaques, an occluded left renal artery, and an occluded coeliac trunk are present. 
Table 1 Results of laboratory tests on three patients with severe systemic atherosclerosis

\begin{tabular}{|c|c|c|c|c|}
\hline Laboratory parameter & Patient 1 & Patient 2 & Patient 3 & Normal values \\
\hline Thrombocyte count $\left(\times 10^{9} / 1\right)$ & 214 & 179 & 336 & $150-350$ \\
\hline \multicolumn{5}{|l|}{ Anticardiolipin antibodies } \\
\hline $\operatorname{Ig} M$ & +++ & - & - & -ve \\
\hline IgG & +++ & ++ & +++ & -ve \\
\hline Lupus anticoagulant & $-\mathrm{ve}$ & $-\mathrm{ve}$ & $-v e$ & $-v e$ \\
\hline Wassermann test & -ve & -ve & $-\mathrm{ve}$ & -ve \\
\hline aPTT (seconds) & 38 & 35 & 29 & $20-40$ \\
\hline Fibrinogen $(\mathrm{g} / \mathrm{l})$ & 6.0 & 5.8 & 3.8 & $2.0-4.0$ \\
\hline Creatinine $(\mu \mathrm{mol} / \mathrm{l})$ & 134 & 85 & 177 & $55-110$ \\
\hline Triglycerides $(\mathrm{mmol} / \mathrm{l})$ & 3.2 & 2.1 & 1.5 & $0.5-2.0$ \\
\hline Cholesterol $(\mathrm{mmol} / \mathrm{l})$ & 4.2 & 6.8 & 3.9 & $4.0-6.5$ \\
\hline HDL cholesterol (mmol/l) & 0.7 & 1.0 & 1.0 & $>1.0$ \\
\hline LDL cholesterol (mmol/l) & 2.1 & 3.7 & 2.3 & $1.68-4.53$ \\
\hline \multicolumn{5}{|l|}{ Vitamins (before treatment) } \\
\hline Vitamin B6 (nmol/1) & 95 & ND & ND & $35-105$ \\
\hline Vitamin B12 (nmol/1) & 0.18 & 0.19 & 0.46 & $0.13-0.60$ \\
\hline Folate $(\mathrm{nmol} / \mathrm{l})$ & 4.1 & 4.1 & 9.9 & $5.0-30.0$ \\
\hline \multicolumn{5}{|l|}{ Methionine loading (I) $(\mu \mathrm{mol} / \mathrm{l})$} \\
\hline Homocysteine (pre/post) & $103 / 159$ & $42 / 78$ & $27 / 94$ & $<15 /<50$ \\
\hline \multicolumn{5}{|c|}{ Methionine loading (II) (after folate treatment) } \\
\hline Homocysteine (pre/post) & $13 / 52$ & $8.6 / 28.6$ & $18 / 52$ & \\
\hline
\end{tabular}

aPTT $=$ activated partial thrombin time; $\mathrm{HDL}=$ high density lipoprotein LDL $=$ low density lipoprotein $\mathrm{ND}=$ not determined.

clinical signs of vascular disease thus far. Apart from weak peripheral pulsations, a physical examination was unremarkable. Angiography showed a normal aorta with occluded iliac arteries on both sides. The common iliac artery showed some distal filling from both hypogastric and inferior mesenteric arteries. Table 1 shows the laboratory data. Hyperhomocysteinaemia was present, and anticardiolipin IgGs were repeatedly found. Testing for lupus anticoagulant was negative. Cholesterol levels were slightly elevated $(6.8 \mathrm{mmol} / \mathrm{l})$. Femoropopliteal bypasses resulted in appreciable improvement in his clinical status. Homocysteine levels normalised after treatment with pyridoxine and folate.

PATIENT 3

This 62 year old woman presented at our outclinic for evaluation of progressive intermittent claudication. These symptoms had been present for eight years. Her smoking history was 20 pack years. There was a family history of myocardial infarction. She had a 10 year history of hypertension and angina pectoris with recent posterior myocardial infarction. Coronary arteriography showed an occluded right coronary artery, with collateral filling, and an $80 \%$ occluded circumflex artery, which was stented after percutaneous transluminal coronary angioplasty. Apart from weak peripheral pulsations and bruits over the abdomen and femoral arteries, a physical examination was unremarkable. Aortic angiography showed a bilateral renal artery stenosis (right side 50\%, left side $70 \%$ ) and considerable aortic atherosclerotic changes with subtotal occlusion of the right common iliac artery. The latter abnormality was treated with balloon dilatation and stent placement. Table 1 gives the laboratory data. As in the other patients, hyperhomocysteinaemia was present, and anticardiolipin IgGs were repeatedly found. Testing for lupus anticoagulant was negative. Homocysteine levels decreased considerably after treatment with pyridoxine/folate, but remained above the normal range. So far, her intermittent claudication has improved considerably.

\section{Discussion}

Renovascular hypertension is most often due to atherosclerosis and fibromuscular dysplasia. ${ }^{12}$ Less common causes include renal artery thrombosis. ${ }^{3}$ One of the causes of arterial thrombosis is the presence of aPLs, including anticardiolipin antibodies. ${ }^{4-7}$ aPLs occur most often in systemic autoimmune diseases, such as systemic lupus erythematosus, ${ }^{4-6}$ and in "primary" antiphospholipid syndrome. ${ }^{68}$

Occlusion of the aorta in the presence of aPLs is rare. ${ }^{610}$ aPLs and deposition of endothelial immune complex have both been suggested to play a role in atherosclerosis in patients with lupus. ${ }^{11-14}$ However, the process of this atherogenicity remains obscure. Premature atherosclerosis of the lower limbs as the first symptom of the antiphospholipid syndrome has been reported. This indicates the possible involvement of aPLs in the pathogenesis of progressive atherosclerosis in these patients. ${ }^{14}$ The concurrent presence of antibodies to low density lipoprotein (anti-LDL) and deposition of LDL/anti-LDL immune complexes with subsequent endothelial damage may also play a role. ${ }^{12}{ }^{13}$ Another possible pathogenic cofactor is dyslipidaemia - that is, the presence of aPLs with increased levels of triglycerides, as found in one of our patients-which has been shown to increase the risk of premature thrombosis. ${ }^{15}$ The probable antigenic target of anticardiolipin antibodies is $\beta_{2}$ glycoprotein $1\left(\beta_{2} \mathrm{GP} 1\right)$, a $50 \mathrm{kDa}$ plasma protein that has anticoagulant effects in vitro. ${ }^{16}$ Anti- $\beta_{2}$ GP1 antibodies may increase the uptake of oxidised LDLs by macrophages, thereby forming foam cells and contributing to atherosclerosis. ${ }^{17}$ In addition, George $e t a l^{18}$ showed a proatherogenic effect of immunisation with $\beta_{2}$ GP1 in a mouse model.

In our patients, none of the other features of "primary" antiphospholipid syndrome or systemic lupus erythematosus were found, whereas several risk factors for atherosclerosis were present. It has been suggested that aPLs may not only be a cause of, but also a sequel to, severe atherosclerosis. The production of aPLs may be triggered by endothelial damage and exposure of antigens to the immune system. ${ }^{19-21}$ Atherosclerotic plaque instability and rupture concurs with local apoptosis of endothelial cells and inflammatory cells such as macrophages and $\mathrm{T}$ lymphocytes. During the late apoptotic process, characteristic changes, called blebbing, occur in the phospholipid phase of the cell membrane. These surface blebs on apoptotic cells show high procoagulant activity and have been associated with the production of aPLs. $^{22} 23$

In addition to the aforementioned factors contributing to atherosclerosis, low HDL cholesterol levels, heavy smoking, and hyperhomocysteinaemia were present in our patients. The latter syndrome has recently been recognised as an important inherited autosomal recessive disorder increasing the risk of atherosclerotic events (reviewed by Boers ${ }^{24}$ ). Hyperhomocysteinaemia is characteristic of homocysteinuria, because of homozygosity for cystathionine synthase deficiency or other rarer enzymatic abnormalities in the methionine-homocysteine 
metabolic pathway. Mild hyperhomocysteinaemia, either during fasting or after a standardised oral methionine load (typically 100 $\mathrm{mg} / \mathrm{kg}$ ), can be the result of intermediate deficiency of one of the enzymes mentioned above. Severe hyperhomocysteinaemia leads to a $50 \%$ chance of vascular problems before the age of 30. Hence, even mild hyperhomocysteinaemia is considered an important risk factor in atherogenesis. Increased homocysteine levels can be reduced by vitamins, including B12, B6, and folate. ${ }^{24}$ In two of our patients, folate levels were slightly decreased (table 1), probably because of an inadequate diet. After vitamin treatment, homocysteine levels decreased considerably. The duration of hyperhomocysteinaemia is not known in our patients. Recent data underline the importance of high homocysteine levels as a risk factor for mortality in patients with coronary artery disease..$^{25}$ Notably, this association was found irrespective of possible coexisting folate deficiency. Accumulating data suggest that homocysteine may affect endothelial resistance to thrombosis. Hyperhomocysteinaemia may thus be an important cofactor in the pathogenesis of the vascular abnormalities in our patients.

The possible association between hyperhomocysteinaemia and the presence of aPLs has been studied previously. ${ }^{26}{ }^{27}$ Although homocysteine levels were higher in lupus patients with renal failure, no association with the presence of aPLs was found. ${ }^{26}$ In another study, aPLs were not found in patients with renal failure and mild hyperhomocysteinaemia. $^{27}$

These cases illustrate that severe (premature) atherosclerosis often results from a spectrum of causes. Established risk factors include hypertension, smoking, and a family history of cardiovascular problems. Hyperhomocysteinaemia and the presence of aPLs should also be considered, because these can be treated with drugs. If aPLs are persistently present (repeated testing), coumarins should be started. A reduction in cardiovascular problems after treatment of hyperhomocysteinaemia remains to be proven (studies are underway). Meanwhile, it seems wise to prescribe folate and pyridoxine for all patients with manifest atherosclerosis. The concurrence of hyperhomocysteinaemia and aPLs in our patients is an interesting finding that merits further research. Whether aPLs in severe atherosclerosis are a cause of the disease and/or a sequel to it remains to be elucidated.

We are indebted to R H W M Derksen, internist, Department of Infectious Diseases and Clinical Immunology, Academic Hospital Utrecht, for critically reading the manuscript.

1 Working group on renovascular hypertension. Detection, evaluation and treatment of renovascular hypertension. Arch Int Med 1987;147:820-9.
2 Stimpel M, Groth H, Greminger P, Luscher TF, Vetter H, Vetter W. The spectrum of renovascular hypertension. Cardiology 1985;72:1-9.

3 Ostuni PA, Lazzarin P, Pengo V, Ruffati A, Schiavon F, Gambari P. Renal artery thrombosis and hypertension in a 13 year old girl with antiphospholipid syndrome. Ann Rheum Dis 1990;49:184-7.

4 Khamashta MA, Wallington T. Management of the antiphospholipid syndrome. Ann Rheum Dis 1991;50: 959-62.

5 Asherson RA, Cervera R. The antiphospholipid syndrome: a syndrome in evolution. Ann Rheum Dis 1992;51:147-50.

6 Asherson RA, Khamashta MA, Ordi-Ros J, Derksen RHWM, Machin SJ, Barquinero J, et al. The 'primary' antiphospholipid syndrome: major clinical and serological features. Medicine 1989;68:366-74.

7 Asherson RA, Derksen RHWM, Harris N, Bingley PJ, Hoffbrand $\mathrm{BI}$, Gharavi AE, et al. Large vessel occlusion and gangrene in systemic lupus erythematosus and 'lupus-like' disease. A report of six cases. J Rheumatol 1986;13:740-7.

8 Asherson RA. A "primary" antiphospholipid syndrome. J Rheumatol 1988;15:1742-6.

9 Alarcón-Segovia D, Sanchez-Guerrero J. Primary antiphospholipid syndrome. J Rheumatol 1989;16:482-8.

10 Alarcón-Segovia D. Pathogenetic potential of antiphospholipid antibodies. J Rheumatol 1988;15:390-3.

11 Baker WF, Bick RL. Antiphospholipid antibodies in coronary artery disease: a review. Semin Thromb Hemost 1994;20:27-45

12 Ames PRJ. Antiphospholipid antibodies, thrombosis and atherosclerosis in systemic lupus erythematosus: a unifying 'membrane stress syndrome' hypothesis. Lupus 1994:3: 371-7.

13 Lopes-Virella MF, Virella G. Atherosclerosis and autoimmunity, short analytical review. Clin Immunol Immunopathol 1994;73:155-67.

14 Levy PJ, Cooper CF, Gonzalez MF. Massive lower extremity arterial thrombosis and acute hepatic insufficiency in a young adult with premature atherosclerosis associated with hyperlipoprotein(a)emia and antiphospholipid syndrome. Ayperlipoprotein(a)emia and

15 Garrido JA, Peromingo JAD, Sesma P, Pia G. More about the link between thrombosis and atherosclerosis in autoimmune diseases: triglycerides and risk for thrombosis in patients with antiphospholipid antibodies. J Rheumatol 1994;21:2394.

16 Alarcon-Segovia D, Cabral AR. The antiphospholipid/ cofactor syndromes. J Rheumatol 1996;23:1319-22.

17 Hasunuma Y, Matsuura E, Makita Z, Katahira T, Nishi S, Kolke T. Involvement of $\beta_{2}$ glycoprotein I and anticardiolipin antibodies in oxidatively modified low density lipoprotein uptake by macrophages. Clin Exp Immunol 1997;107: 569-74.

18 George J, Afek A, Gilburd B, Blank M, Levy Y, Aron-Maor $A$, et al. Induction of early atherosclerosis in LDL-receptordeficient mice immunized with $\beta_{2}$-glycoprotein I. Circulation 1998;98:1108-15

19 Sletnes KE, Smith P, Abdelnoor M, Arnesen H, Wisloff F. Antiphospholipid antibodies after myocardial infarction and their relation to mortality, re-infarction, and nonhaemorrhagic stroke. Lancet 1992;339:451-3.

20 Diaz MN, Becker RC. Anticardiolipin antibodies in patients with unstable angina. Cardiology 1994;84:380-4.

21 Lecerf V, Alhenc-Gelas M, Laurian C, Bruneval P, Aiach M, Cormier JM, et al. Antiphospholipid antibodies and atherosclerosis. Am J Med 1992;92:575-6.

22 Pittoni V, Isenberg D. Apoptosis and antiphospholipid antibodies. Semin Arthritis Rheum 1998;28:163-78.

23 Levine JS, Koh JS, Subang R, Rauch J. Apoptotic cells as immunogen and antigen in the antiphospholipid syndrome. Exp Mol Pathol 1999;66:82-98.

24 Boers GHJ. Hyperhomocysteinaemia: a newly recognized risk factor for vascular disease. Neth J Med 1994;45:34-41.

25 Nygård O, Nordrehaug JE, Refsum H, Ueland PM, Farstad M, Vollset SE. Plasma homocysteine levels and mortality in patients with coronary artery disease. N Engl J Med 1997; 337:230-6.

26 Fijnheer R, Roest M, Haas FJ, de Groot PG, Derksen RH. Homocysteine, methylenetetrahydrofolate reductase polymorphism, antiphospholipid antibodies, and thromboembolic events in systemic lupus erythematosus: a retrospective cohort study. J Rheumatol 1998;25:1737-42.

27 Manns BJ, Burgess ED, Parsons HG, Schaefer JP, Hyndman ME. Hyperhomocysteinemia, anticardiolipin antibody status, and risk for vascular access thrombosis in hemodialysis patients. Kidney Int 1999;55:315-20. 